Review Article

\title{
Cancer Stem Cells: Powerful Targets to Improve Current Anticancer Therapeutics
}

\author{
Rayana L. Bighetti-Trevisan $\mathbb{D}^{1},{ }^{1}$ Lucas O. Sousa $\mathbb{D},{ }^{2}$ Rogerio M. Castilho $\mathbb{D},^{3}$ \\ and Luciana $\mathbf{O}$. Almeida $\mathbb{1}^{1}$ \\ ${ }^{1}$ Laboratory of Tissue Culture, Department of Basic and Oral Biology, University of Sao Paulo School of Dentistry, Ribeirao Preto, \\ SP 14040-904, Brazil \\ ${ }^{2}$ Laboratory of Markers and Cell Signaling of Cancer, Department of Clinical Analyses, Toxicology and Food Sciences, University of \\ Sao Paulo School of Pharmaceutical Sciences, Ribeirao Preto, SP 14040-903, Brazil \\ ${ }^{3}$ Laboratory of Epithelial Biology, Department of Periodontics and Oral Medicine, University of Michigan School of Dentistry, \\ Ann Arbor, MI 48109-1078, USA
}

Correspondence should be addressed to Luciana O. Almeida; lubio2001@usp.br

Received 22 July 2019; Revised 25 September 2019; Accepted 3 October 2019; Published 12 November 2019

Academic Editor: Sumanta Chatterjee

Copyright (C) 2019 Rayana L. Bighetti-Trevisan et al. This is an open access article distributed under the Creative Commons Attribution License, which permits unrestricted use, distribution, and reproduction in any medium, provided the original work is properly cited.

\begin{abstract}
A frequent observation in several malignancies is the development of resistance to therapy that results in frequent tumor relapse and metastasis. Much of the tumor resistance phenotype comes from its heterogeneity that halts the ability of therapeutic agents to eliminate all cancer cells effectively. Tumor heterogeneity is, in part, controlled by cancer stem cells (CSC). CSC may be considered the reservoir of cancer cells as they exhibit properties of self-renewal and plasticity and the capability of reestablishing a heterogeneous tumor cell population. The endowed resistance mechanisms of CSC are mainly attributed to several factors including cellular quiescence, accumulation of ABC transporters, disruption of apoptosis, epigenetic reprogramming, and metabolism. There is a current need to develop new therapeutic drugs capable of targeting CSC to overcome tumor resistance. Emerging in vitro and in vivo studies strongly support the potential benefits of combination therapies capable of targeting cancer stem cell-targeting agents. Clinical trials are still underway to address the pharmacokinetics, safety, and efficacy of combination treatment. This review will address the main characteristics, therapeutic implications, and perspectives of targeting CSC to improve current anticancer therapeutics.
\end{abstract}

\section{Introduction}

Despite the massive amount of research and rapid development of new therapeutic strategies during the past decade, cancer remains a significant public health problem being the second most common cause of death worldwide. It was estimated a total of 18.1 million new cases of cancer in 2018 and 9.6 million deaths worldwide [1].

The carcinogenesis process is driven by a multistep process initiated by the accumulation of successive mutations in normal cells. Despite the extensive efforts in understanding the signaling pathways that control the process of carcinogenesis, and the therapeutic strategies capable of targeting altered signals, the development of new strategies capable of halting cancer progression remains a challenge. Therapy resistance and tumor relapse are frequently observed for most of the malignancies, and they seem to be driven by the cellular heterogeneity that allows drugs to effectively eliminate some, but not all, malignant cells [2].

Malignant tumors are complex systems composed of tumor cells and normal cells of host tissue with different stromal cells, which help to build the phenotypic heterogeneity and malignancy of solid tumors [3]. Intertumor heterogeneity is responsible for the tumor individuality and the difficulty to establish a molecular signature for groups of tumors $[4,5]$. Besides, intratumor heterogeneity presents a distinct molecular signature in every single patient. 
The genetic trail of each tumor directly reflects tumor progression, resistance to therapy, and recurrences damping the efficacy of current therapies [6]. Moreover, tumor heterogeneity is, in part, controlled by a small population of tumor cells presenting self-renewal properties known as cancer stem cells (CSC) [7].

CSC display high metastatic potential and contribute to the resistance to conventional anticancer therapy. CSC are relatively rare tumor cells that can self-renew and give rise to the tumor cell heterogeneity that characterizes the complex architecture of tumors. CSC have been identified in various human cancers such as germ cell cancers [8], leukemia [9], breast cancer [10], brain cancer [11], colon cancer [12], pancreatic cancer [13], melanoma [14], head and neck [15, 16], and several other tumors [17, 18]. The presence of CSC in different tumors suggests a common trend in cancers and thereby a potential target to therapy [19].

The concept of CSC was first introduced in 1928, in which studies recognized similarities among cancer progression and the development of an embryo, originating the embryonic model of tumor origin [20]. However, only in 1991, the CSC model was demonstrated in leukemia, showing the existence of a small population of cells capable of initiating leukemia [9]. Subsequent investigations on different tumors have shown that not all cells in a tumor were endowed with the capacity to propagate efficiently. In fact, it was shown that only CSC have tumorigenic activity that enables them to form tumors when transplanted into animals and can be the source of all tumor cells present in a malignant tumor [21, 22]. It was only later in 2005 that the existence of CSC population was demonstrated in vivo for the first time. Using fully penetrant transgenic mouse models in melanoma [14] and breast [23], intestine [24], and brain cancers [25], researchers identified a group of stem/progenitor cells as cancer-initiating cells and obtained insight into the behavior of these tumors. CSC display resistance to apoptosis, and they are capable of evading the immune system. CSC have similar physiological properties as normal stem cells, like self-renewal, differentiation, and indefinite proliferation ability which might be the main cause of tumor progression [26]. They also can assume a quiescent state, which contributes to the resistance to therapy, and later, they can proliferate and differentiate through asymmetric divisions, promoting recurrence and distant metastases [18].

Current therapies fail to cure metastatic solid tumors; even though they have cytotoxic and/or cytostatic effects over cancer cells, their ability to eliminate cancer stem cells remains poorly understood. The knowledge acquired on CSC biology in recent years supports better detection and isolation and improved therapeutic target of these cells [27]. As a result, the development of new combined therapies, including the use of epigenetic modifiers, stemness inhibitors, and CSC surface markers and immunotherapy are currently in clinical trial [18].

In this review, we will focus on the most recent therapeutic strategies in development targeting CSC and its mechanisms associated with chemo- and radioresistance (Figure 1).

\section{Cancer Stem Cells and Resistance to Therapy}

Probably, one of the challenges of modern medicine is efficiently managing and treating solid tumors. Surgery was the first tool available since 1809 when Ephraim McDowell removed an ovarian tumor providing evidence that some tumors could be cured by surgery. Radiation was developed to fight cancer in 1950. Radiotherapy uses highenergy ionizing radiation to inhibit tumor growth, leading to cell death in some cases $[28,29]$. Advances in radiation therapy lead to marginal success in cancer management with the cure of one-third of all patients receiving combined radiation and surgery [30]. In the early 1900s, Paul Ehrlich started to develop drugs to treat infectious diseases, introducing the term chemotherapy. In cancer, chemotherapy works by killing or slowing down tumors by targeting dividing cells, yet nonproliferating cells are often left behind [31]. The most common drugs, nitrogen mustards, and platinum agents represent more than half of the approximately 4000 open clinical trials using DNA crosslinking agents worldwide. However, only $12 \%$ of these represent late-phase trials focused on relapsed or recurrent cancers [32]. Currently, chemotherapy is the therapy of choice for inoperative tumors and preoperative pharmacotherapy [33]. Some examples of these drugs include temozolomide used as a standard treatment for glioblastoma [34]; docetaxel used to treat metastatic prostate cancer [35]; and platinum derivatives employed in the treatment of several nonoperative cancers such as lung [36], head and neck [37], and colorectal cancers [38]. Combined chemotherapy and radiotherapy have been the therapy of choice for many solid tumors capable of improving overall survival [39].

The population of CSC might be responsible for the lack of success of therapeutic strategies currently available $[40,41]$. Either single or combined treatments target only the bulk of the tumor, and the elevated rates of tumor recurrences are attributed to the accumulation of CSC $[42,43]$. The resistance mechanisms endowed by CSC are attributed to several factors including a transient cellular quiescence, the accumulation of ABC transporters, and disruption of cellular apoptosis [18]. Chemoresistance associated with CSC is observed in colorectal and ovarian cancer, where activation of the serine-threonine kinase named Aurora-A is involved in resistance to apoptosis and tumorigenicity maintenance [44]. In salivary gland tumors, administration of cisplatin is associated with accumulation of CSC and the chemoresistance is controlled by epigenetic modifications $[45,46]$. In glioblastoma, CSC contributes to the resistance to temozolomide through increased expression of the repair protein MGMT, ABC transporter BCRP1, and several antiapoptotic proteins [47].

There is growing evidence that CSC are also innately resistant to radiation, by stimulating the repair of DNA damaged, redistributing the cells in the cell cycle, increasing activation of the DNA damage checkpoint, and repopulating and reoxygenating areas of hypoxia in the tumor [48]. CSC contribute to glioma radioresistance through promptly 

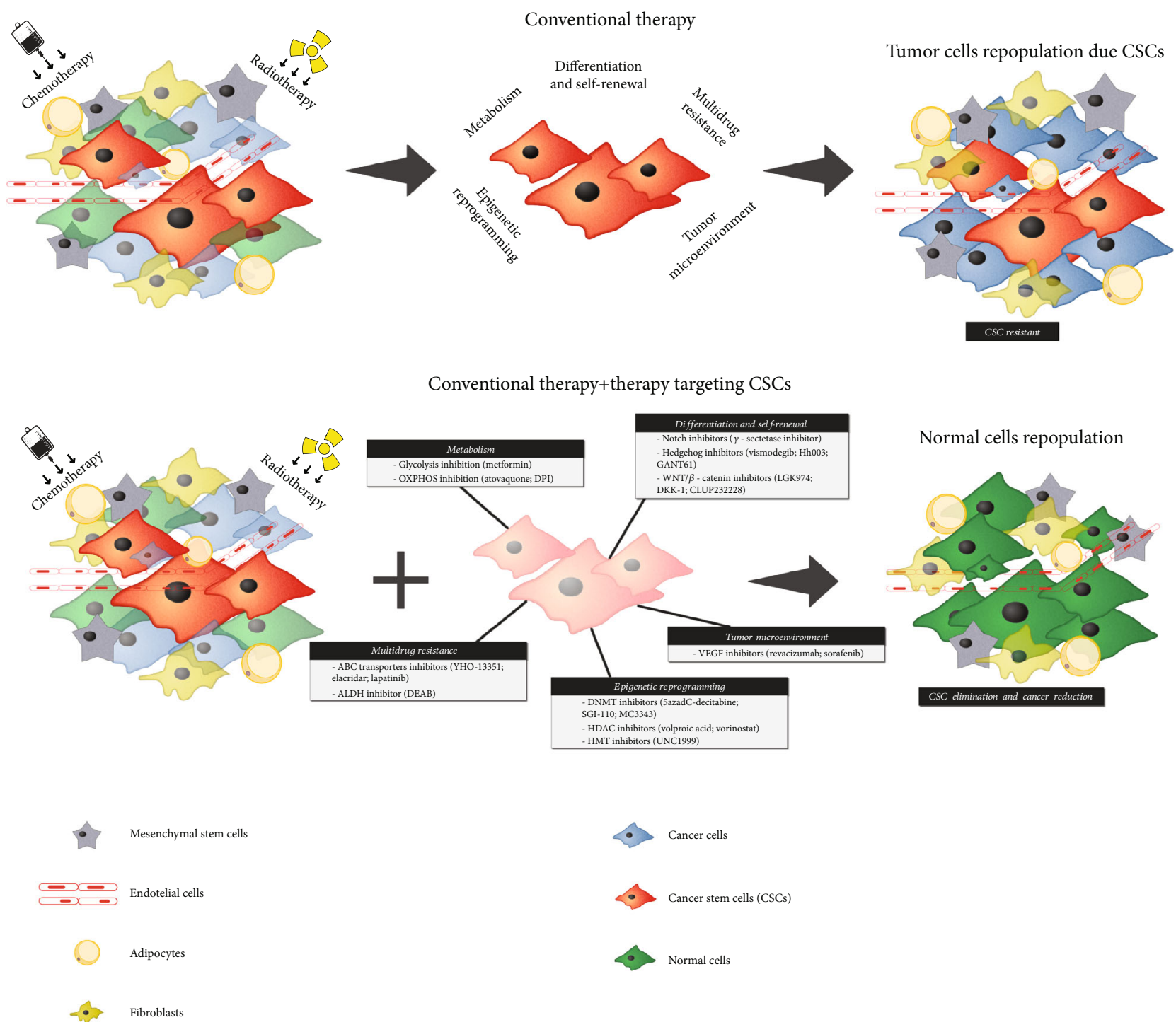

FIGURE 1: Targeting cancer stem cells signaling to overcome resistance of current anticancer therapy. While the conventional chemotherapy and radiotherapy eliminate more differentiated cancer cells, specific phenotypes of CSC (i.e., multidrug resistance, epigenetic reprogramming, and tumor microenvironment protection) allow them to evade the conventional treatments and avoid the cell death. Once CSC population accumulates, after conventional therapy, they start to regrowth the tumor promoting cancer recurrence. To avoid recurrence, a more efficient therapeutic regimen purposes the administration of new drugs that directly target CSC metabolism, self-renewal, differentiation or other stem cell particularities to disrupt CSC, concomitant with conventional therapies to eliminate differentiated cells.

activating DNA damage response, which increases DNA repair, thus promoting cell survival [49]. In breast cancer, radioresistance of CSC is associated with lack of oxidative stress due to their increased ability to eliminate free radicals and the activation of DNA repair [50]. Mammary CSC can also be enriched after radiotherapy through the activation of $\mathrm{WNT} / \beta$-catenin signaling that promotes self-renewal [51]. CSC from mucoepidermoid carcinoma contributes to radioresistance due to the activation of $\mathrm{NF} \kappa \mathrm{B}$ signaling [52]. Overall, the understanding of the role of CSC in tumor formation and maintenance is the key to improve the new therapeutic technologies currently developed to improve short- and long-term survival.

\section{Strategies to Target Cancer Stem Cells}

3.1. Differentiation and Self-Renewal. CSC share many characteristics with normal stem cells. Several studies correlate the origin of CSC with normal stem cells that underwent oncogenic transformation due to mutation-induced genome reprogramming and epigenetic deregulations [53]. The molecular signaling that governs normal stem cell homeostasis is highly regulated. Many of these controlling pathways are abnormally activated leading to the loss of self-renewal and proliferation control, along with increased survival, and differentiation of CSC $[54,55]$. Three main signaling pathways including Notch, Hedgehog, and Wnt sustain survival, 
proliferation, and the balance between differentiation and CSC self-renewal [56].

In normal stem cells, Notch receptor is involved in cell proliferation, differentiation, and apoptosis. Once activated, Notch is translocated to the nucleus, initiating cell transduction and increased transcription. Deregulated Notch signaling leads to abnormal cell proliferation and decreases cellular differentiation and apoptosis and has been implying in the maintenance of CSC in cancer $[57,58]$. The use of Notch inhibitors as a single agent or in combination with chemotherapeutic agents has been applied in the treatment of cancer. Capodanno et al. [59] interfered with the Notch pathway by using gamma-secretase inhibitor (GSI) in combination with 5-fluorouracil (5-FU) and observed a decrease in clonogenicity and tumorigenicity of CSC. In glioblastoma, activation of Notch signaling promotes resistance to temozolomide and radiotherapy, accumulating CSC and activating angiogenesis [60]. The administration of Notch-target gamma-secretase inhibitors in combination with farnesyltransferase inhibitors was efficient in sensitized CSC to radiotherapy [61]. Nanoparticles carrying gamma-secretase inhibitors efficiently reduced self-renewing of CSC and suppressed tumor growth in breast cancer [62].

The Hedgehog $(\mathrm{Hh})$ signaling plays an important role in tissue homeostasis, control of cell polarity, and regulation of embryonic development [63]. Uncontrolled activation of the Hedgehog pathway is associated with many cancers [64-66], and it was recently involved in the chemoresistance phenotype due to the accumulation of CSC $[67,68]$. Small $\mathrm{Hh}$ inhibitors, such as vismodegib (antagonist of smoothened receptor), can suppress cell proliferation and tumor growth [69]. Chen et al. [70] analyzed the effect of Hh003, a new inhibitor of the smoothened receptor (SMO), demonstrating that Hh003 activates caspase-8, inducing apoptosis in colorectal cancer and promoting the inhibition of tumor growth in vivo. The Hh signaling is also related to the maintenance and accumulation of glioma CSC, and the use of the inhibitor diminished proliferation, survival, and self-renewal of CSC, reducing the expression of SOX2, required for stem-cell maintenance [71]. Using a low-throughput drug-screening platform, Balic et al. [72] found that chloroquine efficiently eliminated pancreatic CSC inducing antiproliferative effects via reduction of SMO, improving standard chemotherapeutic regimens. GANT61, a potent inhibitor of the noncanonical Hh pathway reduced CSC in breast cancer cell lines, inhibiting tumor growth through G1 cell cycle arrest and induction of apoptosis [73]. GANT61 also suppressed CSC from pancreatic cancer showing enhanced activity in inhibiting tumor progression when in association with rapamycin [74]. PF-04449913 (PF-913) is a selective, small-molecule inhibitor of SMO. In acute myeloid leukemia (AML), PF913 decreased CSC population, modulating self-renewing properties and cell cycle progression, and it also sensitizes AML to chemotherapy [75].

The Wnt/ $\beta$-catenin pathway is fundamental in the regulation of tissue and stem cell self-renewal [76], but several studies demonstrated the association of $\mathrm{Wnt} / \beta$-catenin and cancer. In the canonical pathway, $\beta$-catenin is translocated to the nucleus, leading to activation of transcription factors that control the expression of target genes. In the noncanonical Wnt pathway, signals are transmitted through GTPase proteins promoting changes in cellular polarity [77]. Inhibition of Wnt signaling using either the porcupine inhibitor LGK974, short hairpin RNA (shRNA) targeting Porcn or recombinant DKK-1 (a Wnt antagonist), reduced CSC, tumor growth, and the proliferative potential of the lung cancer cells, leading to improved survival of patients [78]. Patients with metastatic colorectal cancer have an increased level of blood progastrin, a tumor-promoting peptide essential for self-renewal of colon CSC, which is also a direct target of $\beta$-catenin/TCF4. Antibodies against progastrin efficiently decrease self-renewal of CSC sensitizing colorectal patients to chemotherapy [79]. CWP232228 is a small molecule that potently inhibits Wnt signaling by antagonizing the binding of $\beta$-catenin to T-cell factor (TCF) in the nucleus, which downregulates a subset of $\mathrm{Wnt} / \beta$-catenin-responsive genes. Administration of CWP232228 reduces the accumulation of CSC and reversed radioresistance phenotype in breast cancer [80]. In epithelial ovarian cancer, resistance to platinumbased therapy is associated with the maintenance of CSC via upregulation of the $\mathrm{Wnt} / \beta$-catenin pathway, and the treatment using niclosamide, a salicylamide derivative, promoted significant inhibition of proliferation and eliminated CSC by significantly decreasing the expression of proteins from the Wnt/ $\beta$-catenin pathway [81].

Another pathway involved in the self-renewal and proliferation of normal stem cells and cancer stem cells is the JAK-STAT pathway. Usually, this pathway is activated by several ligands, which phosphorylate and activate JAK following by the recruitment of the transcriptional factor STAT [82]. Nevertheless, in oncogenic situations, the JAK-STAT pathway is unusually activated and contributes to tumor development through the accumulation of CSC. Leng et al. [83] found that the JAK/STAT pathway plays a vital role in the accumulation of glioblastoma stem cells, mediating the resistance to temozolomide and administration of JAK inhibitor AG490, improves the current chemotherapy, inhibiting tumor growth and proliferation of CSC. JAK-STAT signaling is constitutively activated in prostate CSC and blocking STAT3 activation using LLL12, a molecule that inhibits the phosphorylation of STAT3 monomer which suppresses CSC and diminishes tumorigenicity [84]. Celecoxib, a selective COX-2 inhibitor, has been shown to potentially reduce STAT3 phosphorylation. In medulloblastoma, the administration of celecoxib suppressed the CSC-like properties and enhanced the radiotherapy efficiency [85].

3.2. Multidrug Resistance. Among several drug resistance mechanisms used by cancer cells to evade chemotherapy, the ability of tumor cells to increase cellular efflux of administered drugs retaining low intracellular levels is unique. The ATP-binding cassette transporter ( $\mathrm{ABC}$ transporter) is a multidrug efflux pump that can transport substrates and drugs across cellular membranes using ATP hydrolysis. This mechanism is strongly present in CSC which have enhanced anticancer drug efflux, by presenting overactivation of several $\mathrm{ABC}$ transport genes, including $\mathrm{ABCB} 1, \mathrm{ABCG} 2$, and 
ABCC1 [86]. The accumulation of ABCG2 in glioma stem cells (GSC) resistant to demethoxycurcumin (DMC) was evaluated by Chen et al. [87]. It was observed that the suppression of ABCG2 induced apoptosis and increased the levels of reactive oxygen species (ROS) in GSC treated with DMC, overcoming chemoresistance. YHO-13351 is a potent inhibitor of ABCG2, and its combination with irinotecan effectively targets CSC from cervical carcinoma preventing resistance and tumor relapse [88]. Elacridar is a thirdgeneration competitive inhibitor of $\mathrm{ABCB} 1$ that reverses multidrug resistance of lung cancer, sensitizing cancer stem cells to docetaxel [89]. In breast cancer, ABCC1 and ABCC3 transporters are implicated in multidrug resistance and are increased after chemotherapy. Diminished expression of $\mathrm{ABCC} 1$ and $\mathrm{ABCC} 3$ transporters leads to the reduction of CSC population promoting the retention of therapeutic drugs inside cancer cells [90]. Lapatinib is a small molecule that acts at the ATP-binding site of tyrosine kinase domains, and its administration increases the accumulation of chemotherapeutic agents in tumors with multidrug resistance through the inhibition of ABCB1 and ABCG2 in CSC from metastatic breast cancer [91].

ALDH is a major marker of CSC in different cancers. This enzyme is involved in cellular detoxification of normal stem cells through the catalysis of aldophosphamide oxidation and used by CSC to neutralize chemotherapeutic drugs as part of a multidrug resistance process [92]. DEAB (N,Ndiethylaminobenzaldehyde) is a specific ALDH inhibitor that increases the sensitivity of breast cancer CSC presenting high enzymatic levels of ALDH enzymes to paclitaxel and epirubicin [93]. ALDH1A3 is highly expressed in CSC from malignant mesothelioma, and the repression of STAT3-NF $\kappa B$ signaling diminishes ALDH1A3 accumulation sensitizing cancer cells to pemetrexed and cisplatin treatments [94]. ALDH1 activity is also increased in colon cancer stem cells with multidrug resistance phenotype, and the pharmacological inhibition of ALDH1 using DEAB and the molecular inhibition using interference RNA sensitized the cells to capecitabine and 5-fluorouracil [95].

3.3. Epigenetic Reprogramming. Recent advances in gene reprogramming using the Yamanaka factors [96] to induce pluripotency of adult cells suggest that cellular dedifferentiation may play a role in CSC formation development. Moreover, it became clear that cellular reprogramming involves dramatic methyl modifications in $\mathrm{CpG}$ islands [97] supporting the concept that epigenetic modifications may also be involved in dedifferentiation of transformed tumor cells [98]. Indeed, epigenetic reprogramming of primary human colon cancer cells using the expression of OCT4, SOX2, Klf4, and c-MYC generated clonogenic CSC with enhanced metastatic potential. The reprogramming of colon tumor cells reduced the methylation of the promoter region of NANOG leading to protein overexpression. Genetic knockdown of NANOG in the reprogrammed tumor cells eradicated their clonogenic potential. With that, it became evident that epigenetic modifications occurring during cellular reprogramming are critical to the generation of CSC [99].
Since the epigenetic modifications are major events capable of inducing cellular reprogramming and modulating stem cell properties in tumors, the elimination of the CSC can be achieved by targeting epigenetic regulators [100]. JMJD3 is one of two histone H3K27me3 demethylases, and it has been reported to participate in the regulation of tumorigenesis. Upregulation of JMJD3 inhibits the expression of the transcription factor Oct4 promoting the suppression of tumor growth and diminishing CSC from breast cancer, suggesting JMJD3 as a potential target to overcome resistance mediated by CSC [101]. Recent observations on the reduced levels of the Androgen Receptor (AR) in prostate CSC due to the hypermethylation of the promoter region of $\mathrm{AR}$ provided the mechanistic basis to Tian and collaborators [102] to use decitabine (5-aza-2'-deoxycytidine-5azadC) to inhibit DNA methyltransferases (DNMTs) in prostate tumors. This strategy resulted in the elevation of AR levels and the differentiation of the prostate CSC. In breast cancer, low dose of decitabine encapsulated in nanoparticles in combination with doxorubicin efficiently reduced the population of CSC while improving patient response to therapy and accumulation of apoptotic tumor cells [103]. SGI-110 is a secondgeneration DNA methyltransferase inhibitor that reduces the stem-like properties of ovarian cancer cells, such as their tumor-initiating capacity, through the global tumor hypomethylation. SGI-110 was also found to sensitize tumor cells to carboplatin and to reexpress genes associated with differentiation [104]. A recent study [105] described how a new DNMT inhibitor (DNMTi) MC3343 was able to block osteosarcoma cell proliferation. MC3343 presents an antiproliferative effect similar to $5 \mathrm{azadC}$, inducing CSC differentiation and osteoblastic maturation. This drug presents synergistic effects with cisplatin and doxorubicin, and the combination results in DNA damage and cell death.

Another class of epigenetic modifiers used to target CSC is the Histone deacetylases (HDACs) inhibitors. The valproic acid was tested in breast cancer cell line resulting in enhanced acetylation of the $\mathrm{p} 21$ promoter region and increased protein levels of p21. Also, the valproic acid decreased the level of CD44 antigen originating more differentiated cells. The inhibition of HDACs reprogrammed the CSC to a more differentiated phenotype, which is more responsive to the current chemotherapies [106]. Vorinostat (suberoylanilide hydroxamic acid, SAHA) is an efficient class I and II HDAC inhibitor approved in 2006 for the treatment of lymphoma [107]. Vorinostat specifically triggers autophagy and reduces cell viability via differentiation of glioblastoma stem cells. In vivo models revealed that vorinostat effectively reduces tumor growth and induces autophagy through the downregulation of AKT-mTOR signaling [108]. Vorinostat is also effective in reducing CSC from the salivary glands. Administration of vorinostat resulted in the sensitization of adenoid cystic carcinomas and mucoepidermoid carcinomas to cisplatin through the reduction of CSC population [45, 46]. EZH2 is a histone methyltransferase that targets histone $\mathrm{H} 3$ at lysine 9 and 27, leading to transcriptional repression. EZH2 inhibitor UNC1999 efficiently eradicates self-renewal of glioblastoma CSC and reduces tumor growth in combination with temozolomide [109]. 
3.4. CSC Metabolism. Normal proliferating cells under regular oxygen conditions (normoxic) obtain energy to maintain homeostasis converting sequentially glucose to glucose-6phosphate, fructose-6-phosphate, fructose-bisphosphate, and acetyl coenzyme A. In mitochondria, Acetyl CoA enters in the Krebs cycle (or tricarboxylic acid cycle, TCA) to produce nicotinamide adenine dinucleotide (NADH) and flavin adenine dinucleotide (FADH2) molecules. The final balance of OXPHOS generates up to 38 molecules of ATP per glucose molecule. Alternatively, in hypoxic situations, the cytoplasmic glycolytic metabolism is triggered to convert glucose in lactate, generating $\mathrm{NADH}$ and two molecules of ATP per glucose [110]. It is well known that normal stem cells rely their metabolism on glycolysis [111-113] due to their reduced number and immature mitochondria, contributing to an environment that generates less reactive oxygen species (ROS). Interestingly, lower levels of ROS are essential for the undifferentiated stem cell phenotype [114].

3.4.1. CSC Glycolytic Metabolism. In nonsmall lung cancer cell lines, Liu et al. [115] observed that CSC presented higher glycolysis rates than differentiated cells. The level of lactate and the level of genes involved in glycolytic metabolism (such as HK-1, HK-2, Glut-1, and PDK1) were higher in the CSC after glucose administration. CSC from breast tumors also showed a higher glycolytic metabolism when compared to non-CSCs [116]. Undifferentiated breast cancer cells $\left(\mathrm{CD} 49^{\text {high }}\right.$ EPCAM $\left.^{\text {low }}\right)$ presented lower levels of key enzymes involved in the TCA (ldh1, Acol, Sdha, and idh3g) when compared to more differentiated cells (CD49 $9^{\text {lo- }}$ $\left.{ }^{w} E P C A M^{\text {High }}\right)$ [116]. Chen et al. [117] overexpressed NANOG in a mouse model of hepatocellular carcinoma, which led to a decrease in OXPHOS, whereas NANOG knockdown upregulated the OXPHOS genes, reducing glycolytic activity. In oral cancer cells, EGF drives a glucose metabolic reprogramming, and the accumulation of CSC increases the levels of L-lactate through the activation of EGFR/PI3K signaling. The administration of 2-deoxy-Dglucose, a competitive inhibitor of glucose-6-phosphate, efficiently reversed the process diminishing glycolysis and the accumulation of CSC [118]. Metformin, a first-line drug used in the diabetes treatment, was linked to cancer prevention inhibiting cellular transformation and selectively killing breast CSC, which reduces glycolytic and TCA cycle metabolites [119]. In colorectal cancer, metabolic reprogramming of CSC is modulated by the adenylate kinase hCINAP, and the depletion of this enzyme using molecular strategies reverses several CSC phenotypes including selfrenewal, EMT, and chemoresistance [120].

3.4.2. CSC OXPHOS Metabolism. OXPHOS metabolism also contributes to the phenotype of CSC. CD $34^{+}$cells isolated from the bone marrow of patients with Acute Myeloid Leukemia (AML) present low levels of ROS associated with quiescence, self-renewal, and chemotherapy resistance. This CSC (ROS-low) population also presented a lower glycolytic rate when compared to ROS-high population. Still, ROS-low cells were unable to use glycolysis when oligomycin and
FCCP inhibited mitochondrial function, indicating that mitochondrial respiration and function (OXPHOS) is crucial for CSC in AML [121]. In epithelial ovarian cancer (EOC), CSC population $\left(\mathrm{CD} 44^{+} \mathrm{CD} 117^{+}\right)$presented low levels of Phospho-Pyruvate Dehydrogenase (pPDH), Pyruvate Dehydrogenase Kinase (PDHK1), and MCT4 (lactate transporter) when compared to non-CSC. The reduced amount of these enzymes that drive the glycolytic metabolism strongly indicates a commitment to the tricarboxylic acid cycle and OXPHOS metabolism for EOC [122].

Metformin decreases the blood glucose levels through the suppression of hepatic gluconeogenesis and increases glucose uptake by skeletal muscle. Metformin interferes in the mitochondrial respiratory chain, activating $5{ }^{\prime} \mathrm{AMP}$-activated protein kinase (AMPK), suppressing the activity of mTOR, and reducing the levels p-S6K1. This alteration has been shown to sensitize breast CSC $\left(\mathrm{CD} 44^{\mathrm{High}} / \mathrm{CD} 24^{\text {Low }}\right)$ for radiotherapy [123]. Atovaquone is an FDA-approved antimalarial drug and analog of Co-enzyme Q10 (CoQ10) acting as a potent and selective OXPHOS inhibitor, by targeting the CoQ10-dependence of mitochondrial complex III. In breast cancer, atovaquone has anticancer activity against CSC, inhibiting oxygen consumption and accumulating oxidative stress, which induced apoptosis of CSC population [124]. In a high-throughput drug screening, Ozsvari et al. [125] identified DPI (Diphenyleneiodonium chloride), a compound that potently blocks mitochondrial respiration by inhibiting flavin-containing enzymes (FMN and FAD-dependent), as a new potential therapeutic agent against breast CSC. DPI induced a chemoquiescence phenotype that effectively inhibited the propagation of CSC.

3.5. Tumor Microenvironment and CSC. Shelter, perhaps, is the most important function of the tumor microenvironment (TME) for CSC. The TME is composed basically of extracellular matrix, stromal cells (including fibroblasts, mesenchymal, endothelial, and immune cells), and an intricate network of signaling molecules. The whole structure and components of the TME provide ideal conditions for the maintenance of the CSC capacities to unlimited self-renewal, proliferation, differentiation, and generation of the heterogeneous population that characterizes the cancers. Besides, over the past years, this harborage has been implicated in the cancer therapy resistance [126].

3.5.1. Extracellular Matrix. The TME three-dimensional component, noncellular and composed of glycosaminoglycans, collagens, metalloproteases, hyaluronic acid, polysaccharides, glycoproteins, proteoglycans, and a diversity of other proteins, is called extracellular matrix (ECM). Virtually, this well-organized structure is present in all tissues and provides physical stability and an infinitude of signaling that controls cellular survival, proliferation/growth, differentiation, and migration [127].

The balance of all ECM components is closely involved in CSC survival and therapy resistance. One remarkable example of the crosstalk among ECM components and the cancer biology/resistance was described in colorectal liver metastasis. It was observed that the benefits of 
pretreatment of patients with anti-VEGF (bevacizumab) are limited to a few months because of acquired resistance. Metastatic colorectal liver patients and mouse models were treated with anti-VEGF therapy, and the deposition of hyaluronic acid and glycosaminoglycan within the tumors was increased. This situation enhanced the stiffness of the liver tumors, compromising intratumor perfusion and leading to a reduction in intratumor delivery of anticancer drugs [128]. If the tumor mass is not satisfactorily reached by the antitumor drug, the ECM acts as a shield for the CSC. In a hepatocellular carcinoma model, the pharmacological inhibition of hyaluronic acid using 4-methylumbelliferone promoted a decrease in the levels of CSC markers and stimulated apoptosis while decreased tumor growth and metastasis [129].

\subsubsection{Stromal Cells}

(1) Fibroblasts. In a tumor context, the cancer-associated fibroblasts (CAFs) give support to the initiation, development, and maintenance of transformed cells [130]. Nair et al. [131] cultivated mouse-induced stem cells (miPS cells) using conditioned culture medium from two different breast cancer cell lines. The miPS cells differentiated into CSC, presenting high levels of CD133 and epithelial carcinoma marker (EPCAM). These CSC were induced to differentiate and originated myofibroblast-like cells. Myofibroblast-like cells showed a high level of CAF markers (fibroblast-specific protein (FSP1), $\alpha$-smooth muscle actin ( $\alpha$-SMA), stromalderived factor-1 (CXCL12), and transforming growth factor $(\mathrm{TGF} \beta 1)$ ) and were observed that CAFs give support to the CSC self-renewal. In breast, CSC and CAFs interact through Hedgehog signaling, in which CSC secrete SHH ligand regulating CAFs via paracrine activation of Hedgehog. In turn, CAFs secrete a factor that stimulates CSC self-renewal, and the Hedgehog inhibitor vismodegib reduces CAFs and CSC accumulation by slowing tumor formation [132].

(2) Adipocytes. The adipose stem cells (ASCs) are one of the most abundant cells present in the tumor microenvironment. They secrete several molecules with crucial impact in inflammation and angiogenesis, such as leptin, Interleukin-6 (IL-6), and Platelet-derived growth factor (PDGF) [133, 134]. ASC is derived of stem cells from mesenchymal lineage, and they are capable of differentiating into adipocytes, myocytes, osteocytes, or chondrocytes, which draw attention to their therapeutic potential for treating disorders or even their involvement in the development of diseases such as cancer [135]. Conditioned culture media obtained from obese-altered ASC were capable of generating MCF7 breast cancer cell metastasis through the upregulation of ABCB1 and SERPINE1 genes [136]. In prostate cancer, ASC stimulates adipocyte-induced prostate tumor growth through the activation of STAMP2 expression [137]. A recent study demonstrated that coculturing ASC pretreated with paclitaxel inhibited breast cancer proliferation and survival in vitro and in vivo, indicating the potential of this strategy to avoid cancer relapse [138]. Regarding CSC, adipocytes from prostate cancer niche induce CSC accumulation through the stimulation of paracrine factors such as cathepsin B [139].

(3) Mesenchymal Cells. Mesenchymal stem cells (MSCs), first isolated from the bone marrow and found in several other tissues, present the plasticity in differentiating into the cartilage, bone, and fat cells, among others [140]. It has been described in TME and in the neoplastic niches that MSC support cancer cell proliferation, protect them against ROS, and stimulate the epithelial-mesenchymal transition (EMT), inducing transformed cells to get more metastatic phenotype [141]. MSC in TME is also associated with resistance to chemotherapy. Using two different mouse tumor models (colon carcinoma and Lewis lung carcinoma), Roodhart et al. [142] observed that the intravenous injection of MSC induced resistance to various chemotherapeutics and this MSC-induced resistance was dependent on the activation of the mesenchymal cells with platinum-based drug.

(4) Endothelial Cells. As basic vasculature units, endothelial cells (EC) have the function not only as structural entities, giving rise to the system that provides oxygen and nutrients to the tumors. EC also work as mediators of signaling in the TME, contributing to survival, self-renewal, EMT, and metastatic phenotype of the cancer cells [143]. It has been observed that the endothelial nitric oxide synthase (eNOS) expression and activity are higher in glioma cells neighboring the vascular endothelium. In this sense, Charles et al. [144] described that Nitric Oxide (NO) activates the Notch pathway in glioma stem cells located close to the endothelium and this activation of Notch signaling accelerates the onset of glioma and tumor formation in murine model increasing CSC.

3.5.3. Immune Cells. Failures of the immune system to identify and eliminate transformed cells have been described as a major cause for cancer development [145]. CSC can modify the immune cells located in the TME to maintain an enabling environment. This situation was observed by studying CSC in a rat glioma model. The authors observed that CSC induced bone marrow-derived monocytes to differentiate into tumor-associated macrophages (TAM). The TAM $\mathrm{CD} 11 \mathrm{c}^{\text {high }}$ showed protumor activity, and its generation was attributed to the secretion of GM-CSF by the CSC, putting TMA CD11c $\mathrm{c}^{\text {high }}$ as a candidate for therapy to destabilize CSC niches [146]. Immunologic malfunction also occurs because CSC acquires abilities to escape from the immune system. After $\mathrm{CD} 8^{+} \mathrm{T}$ cells were incubated with CSC $\left(\mathrm{CD} 44^{+}\right)$or more differentiated cells $\left(\mathrm{CD} 44^{-}\right)$ isolated from head and neck squamous cell carcinoma (HNSCC), the authors observed that the $\mathrm{CD}^{+} \mathrm{T}$ cells stimulated with $\mathrm{CD} 44^{+}$cells produced less IFN $\gamma$ when compared to CD44, showing that CSC present less immunogenicity and this feature favors a scape from the immunologic system [147]. Understanding the molecular mechanisms that lead CSC to escape from the immune system is perhaps one of the most important tasks to be done in the study of cancer. 
TABLE 1: Clinical trials involving combination treatment targeting cancer stem cells to overcome chemo- and radioresistance.

\begin{tabular}{|c|c|c|}
\hline Tumor type & Clinical trial ID & Drug targeting CSC (plus chemo/radiotherapy) \\
\hline Breast & $\begin{array}{c}\text { NCT02876302; NCT02776917; NCT02370238; } \\
\text { NCT01868503; NCT01876251; NCT02001974; } \\
\text { NCT01372579; NCT01281163; NCT01190345; } \\
\text { NCT01118975; NCT00949247; NCT00645333; } \\
\text { NCT00524303 }\end{array}$ & $\begin{array}{l}\text { Ruxolitinib; cirmtuzumab; reparixin; lapatinib; PF- } \\
\text { 03084014; eribulin mesylate; MK2206; bevacizumab; } \\
\text { vorinostat; MK0752; trastuzumab }\end{array}$ \\
\hline Pancreatic & $\begin{array}{l}\text { NCT022311723; NCT01195415; NCT01192763; } \\
\text { NCT01189929; NCT01051284 }\end{array}$ & $\begin{array}{c}\text { BBI608; vismodegib; RO4929097; demcizumab; cyberknife } \\
\text { radiation }\end{array}$ \\
\hline Ovarian & $\begin{array}{l}\text { NCT03632798; NCT03030287; NCT02713386; } \\
\text { NCT01579812 }\end{array}$ & Avastin; OMP-305B83; ruxolitinib; metformin \\
\hline Hematologic & $\begin{array}{l}\text { NCT03113643; NCT02730195; NCT01861340; } \\
\text { NCT01130688 }\end{array}$ & Azacitidine; pioglitazone; MEDI-551; zileuton \\
\hline Glioma & $\begin{array}{l}\text { NCT03632135; NCT02315534; NCT02039778; } \\
\text { NCT01119599 }\end{array}$ & $\begin{array}{l}\text { Vincristine; irinotecan; etoposide; imatinib; BBI608; stem } \\
\text { cell radiotherapy; RO4929097 }\end{array}$ \\
\hline Colorectal & NCT03035253; NCT02753127; NCT01189942 & OMP-305B83; BBI608; OMP-21M18 \\
\hline Non-small-cell lung & NCT01193868; NCT01189968 & RO4929097; demcizumab \\
\hline Hepatocellular & NCT02279719; NCT01442870 & BBI503; BBI608; metformin \\
\hline Esophageal & NCT02423811 & Fursultiamine \\
\hline Gastrointestinal & NCT02024607 & BBI608 \\
\hline Head and neck & NCT01255800 & IPI-926 \\
\hline Advanced or metastatic & $\begin{array}{l}\text { NCT02722954; NCT02483247; NCT02467361; } \\
\text { NCT02432326 }\end{array}$ & Demcizumab; BBI503; BBI608 \\
\hline
\end{tabular}

\section{Combination Treatment as a Strategy to Eliminate CSC and Reverse Chemo- and Radioresistance}

After diagnosis, a tumor harbors at least ten or more subclones that are resistant to monotherapy. Consequently, single-targeted agents are unlikely to effectively kill all types of tumor cells due to tumor heterogeneity [148]. Besides, many tumors develop resistance post treatment, urging the discovery of novel therapeutics. Furthermore, identifying agents that can be administrated in combination is a strategy to avoid or overcome treatment failure by minimizing resistance and preventing further progression to metastatic disease [32].

The accumulation of data from cancer cell lines and animal models strongly supports the potential benefits of combination treatment. Several clinical trials are currently under investigation to explore efficacy, safety, and pharmacokinetics of combining chemotherapeutic drugs with anti-CSC agents. A summary of clinical trials using combination treatment is displayed in Table 1 (according to the United States National Cancer Institute ClinicalTrials.gov). NCT01876251 is a phase I clinical trial that evaluates maximum tolerated dose, safety, pharmacokinetics, and antitumor activity of the combination between PF-03084014 and docetaxel in patients with breast cancer. $\mathrm{PF}-03084014$ is a reversible, non-competitive, selective gamma-secretase inhibitor that blocks NOTCH signaling, interfering with the survival of CSC. Sixteen percent of the patients present a partial response to the treatment [149]. A successful trial evaluating the inhibition of NOTCH signaling to target CSC is in phase IB (NCT01189968) and associates demcizumab combined with carboplatin and pemetrexed as first-line treatment for patients with previously untreated advanced-stage nonsquamous non-small-cell lung cancer (NSCLC). Demcizumab is an IgG2 humanized monoclonal antibody directed against delta-like ligand 4-Notch (DLL4Notch) signaling, which contributes to chemoresistant CSC and tumor vasculature. The primary objective of this trial was to determine the maximum tolerated dose of the combination and define safety, rates of immunogenicity, preliminary efficacy, pharmacokinetics, and pharmacodynamics. Fifty percent of the patients evaluated had objective tumor responses, which was numerically higher than the expected with the standard pemetrexed-platinum chemotherapy, and the combination was recommended to phase II study [150]. Another successful clinical trial targets CXCR1, which is an actionable receptor selectively expressed by breast cancer stem cells (BCSC). CXCR1 is a receptor for CXC ligand 8 (CXCL8; formerly interleukin 8), a proinflammatory chemokine implicated in metastasis and progression of multiple malignancies. Reparixin is an allosteric inhibitor of CXCR1/2 that demonstrates activity against BCSC in xenograft models. A phase IB clinical trial examined dose, safety, and pharmacokinetics of paclitaxel plus reparixin therapy, and explored effects of reparixin on BCSCs in metastatic breast cancer patients (NCT02001974). The combination seemed to be safe and tolerable, and a $30 \%$ response rate was recorded with a reduction of paclitaxel doses, suggesting further studies in a randomized phase II trial [151].

Despite the fact that CSC are resistant to radiotherapy $[39,152]$ and are often found accumulated after radiotherapy $[153,154]$, few clinical trials focus on targeting CSC to 
overcome radioresistance. NCT02039778 was a phase II study focusing on targeting the CSC niche that resides in the lateral periventricular regions of the brain, using irradiation combined to temozolomide in patients with high-grade glioma. Another phase II study aimed to combine fursultiamine plus irradiation and chemotherapy (NCT02423811). Fursultiamine (also known as thiamine tetrahydrofurfuryl disulfide, TTFD) is a derivative of vitamin $\mathrm{B}$ used as a nutrition supplement that efficiently suppresses CSC markers OCT-4, SOX-2, and NANOG expression and decreased $\mathrm{ABCB} 1$ and $\mathrm{ABCG} 2$ in tumor spheres of esophageal carcinoma. NCT01119599 is a phase I trial of RO4929097 (gamma-secretase/Notch signaling pathway inhibitor) in combination with standard radiotherapy and temozolomide for newly diagnosed malignant glioma. Unfortunately, none of these studies have provided significant results yet (source: United States National Cancer Institute ClinicalTrials.gov).

Besides the growing amount of clinical trials ongoing, several preclinical studies are currently investigating new approaches to target CSC and reverse resistance. CBL0137 is an inhibitor of FACT (histone chaperone facilitates chromatin transcription) highly expressed in CSC. De et al. [155] explored the ability to combine cisplatin and CBL0137 to target small cell lung CSC and to reduce tumor size. CBL0137 alone efficiently diminished CSC, and the combination of cisplatin and CBL0137 significantly reduced the growth of PDX tumor models and also the growth of a syngeneic mouse SCLC tumor. BBI608 is a small molecule that inhibits STAT3, a critical mediator for the maintenance of cancer stemness. BBI608 suppresses cancer relapse, progression, and metastasis. BBI608 inhibited stemness gene expression, depleted CSC, and overcame cisplatin resistance in NSCLC [156]. Cell surface vimentin (CSV) is an effective target to reduce CSC. Unlike intracellular vimentin, which is found in both cancer cells and normal mesenchymal cells, CSV is tumor-specific and is accumulated in CSC from glioblastoma. A novel monoclonal antibody 86C, which recognizes CSV from cancer stem cells, induces apoptosis and overcomes temozolomide resistance in glioblastoma, increasing cell death. Eighty-six $\mathrm{C}$ administration reduced the dose of temozolomide required to eliminate tumor cells reducing toxicity [157].

Regarding radioresistance, ongoing preclinical studies have been exploring DNA damage response and apoptosis signaling of CSC to sensitize these cells to radiotherapy. For instance, Timme et al. [158] evaluated the small molecule VX-984 as a radiosensitizer of glioblastoma CSC. VX-984 is an inhibitor of the phosphorylation of DNA-PK, an essential component of the NHEJ repair pathway. VX-984 significantly enhanced the antitumor effect of irradiation in glioblastoma CSC and orthotopic GBM models. PARP protein detects the presence of DNA damage and activates signaling pathways that promote appropriate cellular responses. Thus, PARP inhibitors (PARPi) have emerged as potential radiosensitizing agents. Lesueur et al. [159] investigated the preclinical efficacy of talazoparib, a new PARPi, in association with radiation in reducing CSC population of glioblastomas. The combination drastically reduced accumulation of CSC in vitro. Furthermore, talazoparib combined with irradiation induced a prolonged G2/M block decreasing proliferation.
Afatinib is a pan-EGFR inhibitor that radiosensitizes head and neck squamous cell carcinoma blocking the accumulation of CSC, inhibiting DNA repair machinery, and inducing apoptosis. Combination of afatinib plus irradiation efficiently decreased the tumor volume in xenograft models [160].

\section{Conclusions}

The journey of cancer therapy is far from the end, and major efforts are necessary to develop effective treatments. CSC population has been presenting resistance to most chemotherapy and radiotherapy regimens currently in use. CSC are continually evolving, constituting functionally heterogeneous cells that are maintained by the plasticity of its niche. Besides chemo- and radioresistance, CSC are also responsible for cancer initiation, tumor relapse, and metastasis, emerging as the major target for the development of successful therapies. Molecular targets that control CSC have the potential to drive the development of new drugs capable of eradicating and preventing the accumulation of new CSC in patients, which could prevent metastasis and tumor relapse, reducing morbidity and toxicity, and ultimately improving the outcomes in cancer patients. Currently, several cell and animal studies strongly support the potential benefits of combining chemo/radiotherapy with CSC-targeting agents; however, the effectiveness of these strategies to improve therapeutic regimens for resistant cancer patients remains largely unknown. Still, a myriad of CSC-targeting agents have been investigated in preclinical and clinical studies, and as more studies are conducted and completed, we expect to achieve a better understanding about the safety and efficacy of the combination strategies.

\section{Disclosure}

The funders had no role in study design, data collection, data analysis, decision to publish, or preparation of the paper.

\section{Conflicts of Interest}

The authors declare that they have no conflict of interest.

\section{Acknowledgments}

This study was supported by the Sao Paulo Research Foundation (FAPESP) grant numbers 2017/11780-8 and 2018/02959-7. The authors thank Apparecido Trevisan Junior for the graphic design of Figure 1.

\section{References}

[1] J. Ferlay, M. Colombet, I. Soerjomataram et al., "Estimating the global cancer incidence and mortality in 2018: GLOBOCAN sources and methods," International Journal of Cancer, vol. 144, no. 8, pp. 1941-1953, 2019.

[2] N. K. Lytle, A. G. Barber, and T. Reya, "Stem cell fate in cancer growth, progression and therapy resistance," Nature Reviews Cancer, vol. 18, no. 11, pp. 669-680, 2018.

[3] A. Kreso and J. E. Dick, "Evolution of the cancer stem cell model," Cell Stem Cell, vol. 14, no. 3, pp. 275-291, 2014. 
[4] M. Uhlen, C. Zhang, S. Lee et al., "A pathology atlas of the human cancer transcriptome," Science, vol. 357, no. 6352, article eaan2507, 2017.

[5] L. D. Wood, D. W. Parsons, S. Jones et al., "The genomic landscapes of human breast and colorectal cancers," Science, vol. 318, no. 5853, pp. 1108-1113, 2007.

[6] G. Stanta and S. Bonin, "Overview on clinical relevance of intra-tumor heterogeneity," Frontiers in Medicine, vol. 5, p. 85, 2018.

[7] L. V. Nguyen, R. Vanner, P. Dirks, and C. J. Eaves, "Cancer stem cells: an evolving concept," Nature Reviews Cancer, vol. 12, no. 2, pp. 133-143, 2012.

[8] S. A. Bapat, A. M. Mali, C. B. Koppikar, and N. K. Kurrey, "Stem and progenitor-like cells contribute to the aggressive behavior of human epithelial ovarian cancer," Cancer Research, vol. 65, no. 8, pp. 3025-3029, 2005.

[9] L. W. Terstappen, S. Huang, M. Safford, P. M. Lansdorp, and M. R. Loken, "Sequential generations of hematopoietic colonies derived from single nonlineage-committed CD34 +CD38- progenitor cells," Blood, vol. 77, no. 6, pp. 12181227, 1991.

[10] M. Al-Hajj, M. S. Wicha, A. Benito-Hernandez, S. J. Morrison, and M. F. Clarke, "Prospective identification of tumorigenic breast cancer cells," Proceedings of the National Academy of Sciences of the United States of America, vol. 100, no. 7, pp. 3983-3988, 2003.

[11] S. K. Singh, I. D. Clarke, M. Terasaki et al., "Identification of a cancer stem cell in human brain tumors," Cancer Research, vol. 63, no. 18, pp. 5821-5828, 2003.

[12] C. A. O’Brien, A. Pollett, S. Gallinger, and J. E. Dick, “A human colon cancer cell capable of initiating tumour growth in immunodeficient mice," Nature, vol. 445, no. 7123, pp. 106-110, 2007.

[13] C. Li, D. G. Heidt, P. Dalerba et al., "Identification of pancreatic cancer stem cells," Cancer Research, vol. 67, no. 3, pp. 1030-1037, 2007.

[14] D. Fang, T. K. Nguyen, K. Leishear et al., "A tumorigenic subpopulation with stem cell properties in melanomas," Cancer Research, vol. 65, no. 20, pp. 9328-9337, 2005.

[15] M. E. Prince, R. Sivanandan, A. Kaczorowski et al., "Identification of a subpopulation of cells with cancer stem cell properties in head and neck squamous cell carcinoma," Proceedings of the National Academy of Sciences of the United States of America, vol. 104, no. 3, pp. 973-978, 2007.

[16] L. O. Almeida, D. M. Guimaraes, C. H. Squarize, and R. M. Castilho, "Profiling the behavior of distinct populations of head and neck cancer stem cells," Cancers, vol. 8, no. 1, 2016.

[17] I. Baccelli and A. Trumpp, "The evolving concept of cancer and metastasis stem cells," The Journal of Cell Biology, vol. 198, no. 3, pp. 281-293, 2012.

[18] S. Talukdar, L. Emdad, S. K. Das, D. Sarkar, and P. B. Fisher, "Evolving strategies for therapeutically targeting cancer stem cells," Advances in Cancer Research, vol. 131, pp. 159-191, 2016.

[19] Y. Zhao, Q. Dong, J. Li et al., "Targeting cancer stem cells and their niche: perspectives for future therapeutic targets and strategies," Seminars in Cancer Biology, vol. 53, pp. 139-155, 2018.

[20] E. T. Krebs, "Cancer and the embryonal hypothesis," California Medicine, vol. 66, no. 4, pp. 270-271, 1947.
[21] T. Reya, S. J. Morrison, M. F. Clarke, and I. L. Weissman, "Stem cells, cancer, and cancer stem cells," Nature, vol. 414, no. 6859, pp. 105-111, 2001.

[22] M. F. Clarke, J. E. Dick, P. B. Dirks et al., "Cancer stem cell$\mathrm{s}$-perspectives on current status and future directions: AACR Workshop on cancer stem cells," Cancer Research, vol. 66, no. 19, pp. 9339-9344, 2006.

[23] R. W. Cho, X. Wang, M. Diehn et al., "Isolation and Molecular Characterization of Cancer Stem Cells in MMTV-Wnt-1 Murine Breast Tumors," Stem Cells, vol. 26, no. 2, pp. 364371, 2008.

[24] N. Barker, R. A. Ridgway, J. H. van Es et al., "Crypt stem cells as the cells-of-origin of intestinal cancer," Nature, vol. 457, no. 7229, pp. 608-611, 2009.

[25] S. Alcantara Llaguno, J. Chen, C. H. Kwon et al., "Malignant astrocytomas originate from neural stem/progenitor cells in a somatic tumor suppressor mouse model," Cancer Cell, vol. 15, no. 1, pp. 45-56, 2009.

[26] D. Bonnet and J. E. Dick, "Human acute myeloid leukemia is organized as a hierarchy that originates from a primitive hematopoietic cell," Nature Medicine, vol. 3, no. 7, pp. 730737, 1997.

[27] S. Dawood, L. Austin, and M. Cristofanilli, "Cancer stem cells: implications for cancer therapy," Oncology, vol. 28, no. 12, pp. 1101-1107, 2014.

[28] A. C. Parplys, E. Petermann, C. Petersen, E. Dikomey, and K. Borgmann, "DNA damage by X-rays and their impact on replication processes," Radiotherapy and Oncology, vol. 102, no. 3, pp. 466-471, 2012.

[29] T. I. Liu, Y. C. Yang, W. H. Chiang et al., "Radiotherapy-controllable chemotherapy from reactive oxygen speciesresponsive polymeric nanoparticles for effective local dual modality treatment of malignant tumors," Biomacromolecules, vol. 19, no. 9, pp. 3825-3839, 2018.

[30] V. T. DeVita Jr. and S. A. Rosenberg, "Two hundred years of cancer research," The New England Journal of Medicine, vol. 366, no. 23, pp. 2207-2214, 2012.

[31] I. Masood, M. H. Kiani, M. Ahmad, M. I. Masood, and H. Sadaquat, "Major contributions towards finding a cure for cancer through chemotherapy: a historical review," Tumori, vol. 102, no. 1, pp. 6-17, 2016.

[32] E. Yeh, "The continuing evolution of strategies for cancer therapeutics," Current Opinion in Pharmacology, vol. 41, pp. iv-vi, 2018.

[33] Y. Sumikawa, S. Inui, Y. Nishimura, E. Morita, and K. Kurachi, "Long-term metronomic docetaxel chemotherapy for inoperative angiosarcoma of the scalp," The Journal of Dermatology, vol. 38, no. 4, pp. 393-395, 2011.

[34] F. Saran, O. L. Chinot, R. Henriksson et al., "Bevacizumab, temozolomide, and radiotherapy for newly diagnosed glioblastoma: comprehensive safety results during and after first-line therapy," Neuro-Oncology, vol. 18, no. 7, pp. 9911001, 2016.

[35] N. D. James, M. R. Sydes, N. W. Clarke et al., "Addition of docetaxel, zoledronic acid, or both to first-line long-term hormone therapy in prostate cancer (STAMPEDE): survival results from an adaptive, multiarm, multistage, platform randomised controlled trial," The Lancet, vol. 387, no. 10024, pp. 1163-1177, 2016.

[36] C. Gridelli, F. Ciardiello, C. Gallo et al., "First-line erlotinib followed by second-line cisplatin-gemcitabine chemotherapy 
in advanced non-small-cell lung cancer: the torch randomized trial," Journal of Clinical Oncology, vol. 30, no. 24, pp. 3002-3011, 2012.

[37] J. B. Vermorken, R. Mesia, F. Rivera et al., "Platinum-based chemotherapy plus cetuximab in head and neck cancer," The New England Journal of Medicine, vol. 359, no. 11, pp. 1116-1127, 2008.

[38] M. H. Rasmussen, N. F. Jensen, L. S. Tarpgaard et al., "High expression of microRNA-625-3p is associated with poor response to first-line oxaliplatin based treatment of metastatic colorectal cancer," Molecular Oncology, vol. 7, no. 3, pp. 637-646, 2013.

[39] G. Yoon, S. M. Kim, H. J. Kim, and A. N. Seo, "Clinical influence of cancer stem cells on residual disease after preoperative chemoradiotherapy for rectal cancer," Tumour Biology, vol. 37, no. 3, pp. 3571-3580, 2016.

[40] A. Nogueira-Rodrigues, G. Moralez, R. Grazziotin et al., "Phase 2 trial of erlotinib combined with cisplatin and radiotherapy in patients with locally advanced cervical cancer," Cancer, vol. 120, no. 8, pp. 1187-1193, 2014.

[41] Y. Lin, F. Kong, H. Li et al., "Comparison of target volume and clinical effects of four radiotherapy plans for acute lymphoblastic leukemia prior to hematopoietic stem cell transplantation," Molecular Medicine Reports, vol. 18, no. 3, pp. 2762-2770, 2018.

[42] G. Szakacs, J. K. Paterson, J. A. Ludwig, C. Booth-Genthe, and M. M. Gottesman, "Targeting multidrug resistance in cancer," Nature Reviews Drug Discovery, vol. 5, no. 3, pp. 219234, 2006.

[43] S. Badve and H. Nakshatri, "Breast-cancer stem cells-beyond semantics," The Lancet Oncology, vol. 13, no. 1, pp. e43-e48, 2012.

[44] P. Cammareri, A. Scopelliti, M. Todaro et al., "Aurora-a is essential for the tumorigenic capacity and chemoresistance of colorectal cancer stem cells," Cancer Research, vol. 70, no. 11, pp. 4655-4665, 2010.

[45] L. O. Almeida, D. M. Guimaraes, M. D. Martins et al., "Unlocking the chromatin of adenoid cystic carcinomas using HDAC inhibitors sensitize cancer stem cells to cisplatin and induces tumor senescence," Stem Cell Research, vol. 21, pp. 94-105, 2017.

[46] D. M. Guimaraes, L. O. Almeida, M. D. Martins et al., "Sensitizing mucoepidermoid carcinomas to chemotherapy by targeted disruption of cancer stem cells," Oncotarget, vol. 7, no. 27, pp. 42447-42460, 2016.

[47] G. Liu, X. Yuan, Z. Zeng et al., "Analysis of gene expression and chemoresistance of CD133+ cancer stem cells in glioblastoma," Mol Cancer, vol. 5, article 67, 2006.

[48] F. Pajonk, E. Vlashi, and W. H. McBride, "Radiation Resistance of Cancer Stem Cells: The 4 R's of Radiobiology Revisited," Stem Cells, vol. 28, no. 4, pp. 639-648, 2010.

[49] S. Bao, Q. Wu, R. E. McLendon et al., "Glioma stem cells promote radioresistance by preferential activation of the DNA damage response," Nature, vol. 444, no. 7120, pp. 756-760, 2006.

[50] T. M. Phillips, W. H. McBride, and F. Pajonk, "The response of $\mathrm{CD} 24^{- \text {llow }} / \mathrm{CD} 44^{+}$breast cancer-initiating cells to radiation," Journal of the National Cancer Institute, vol. 98, no. 24, pp. 1777-1785, 2006.

[51] W. A. Woodward, M. S. Chen, F. Behbod, M. P. Alfaro, T. A. Buchholz, and J. M. Rosen, "WNT/ $\beta$-catenin medi- ates radiation resistance of mouse mammary progenitor cells," Proceedings of the National Academy of Sciences of the United States of America, vol. 104, no. 2, pp. 618-623, 2007.

[52] V. P. Wagner, M. A. Martins, M. D. Martins et al., "Overcoming adaptive resistance in mucoepidermoid carcinoma through inhibition of the IKK- $\beta / \mathrm{I} \kappa \mathrm{B} \alpha / \mathrm{NF} \kappa \mathrm{B}$ axis," Oncotarget, vol. 7, no. 45, pp. 73032-73044, 2016.

[53] J. E. Visvader, "Cells of origin in cancer," Nature, vol. 469, no. 7330, pp. 314-322, 2011.

[54] M. E. Issa, F. S. Takhsha, C. S. Chirumamilla, C. Perez-Novo, W. Vanden Berghe, and M. Cuendet, "Epigenetic strategies to reverse drug resistance in heterogeneous multiple myeloma," Clinical Epigenetics, vol. 9, article 17, 2017.

[55] E. M. De Francesco, F. Sotgia, and M. P. Lisanti, "Cancer stem cells (CSCs): metabolic strategies for their identification and eradication," Biochemical Journal, vol. 475, no. 9, pp. 1611-1634, 2018.

[56] B. Lu, X. Huang, J. Mo, and W. Zhao, "Drug delivery using nanoparticles for cancer stem-like cell targeting," Frontiers in Pharmacology, vol. 7, p. 84, 2016.

[57] P. Rizzo, C. Osipo, K. Foreman, T. Golde, B. Osborne, and L. Miele, "Rational targeting of Notch signaling in cancer," Oncogene, vol. 27, no. 38, pp. 5124-5131, 2008.

[58] J. N. Zhu, L. Jiang, J. H. Jiang et al., "Hepatocyte nuclear factor-1beta enhances the stemness of hepatocellular carcinoma cells through activation of the Notch pathway," Scientific Reports, vol. 7, no. 1, article 4793, 2017.

[59] Y. Capodanno, F. O. Buishand, L. Y. Pang, J. Kirpensteijn, J. A. Mol, and D. J. Argyle, "Notch pathway inhibition targets chemoresistant insulinoma cancer stem cells," EndocrineRelated Cancer, vol. 25, no. 2, pp. 131-144, 2018.

[60] C. A. Gilbert, M. C. Daou, R. P. Moser, and A. H. Ross, "Gamma-secretase inhibitors enhance temozolomide treatment of human gliomas by inhibiting neurosphere repopulation and xenograft recurrence," Cancer Research, vol. 70, no. 17, pp. 6870-6879, 2010.

[61] Y. Ma, Z. Cheng, J. Liu et al., "Inhibition of farnesyltransferase potentiates NOTCH-targeted therapy against glioblastoma stem cells," Stem Cell Reports, vol. 9, no. 6, pp. 1948-1960, 2017.

[62] V. Mamaeva, R. Niemi, M. Beck et al., "Inhibiting Notch Activity in Breast Cancer Stem Cells by Glucose Functionalized Nanoparticles Carrying $\gamma$-secretase Inhibitors," Molecular Therapy, vol. 24, no. 5, pp. 926-936, 2016.

[63] N. Takebe, P. J. Harris, R. Q. Warren, and S. P. Ivy, "Targeting cancer stem cells by inhibiting Wnt, Notch, and Hedgehog pathways," Nature Reviews Clinical Oncology, vol. 8, no. 2, pp. 97-106, 2011.

[64] M. Hui, A. Cazet, R. Nair, D. N. Watkins, S. A. O'Toole, and A. Swarbrick, "The Hedgehog signalling pathway in breast development, carcinogenesis and cancer therapy," Breast Cancer Research, vol. 15, no. 2, p. 203, 2013.

[65] N. Li, S. Truong, M. Nouri et al., "Non-canonical activation of hedgehog in prostate cancer cells mediated by the interaction of transcriptionally active androgen receptor proteins with Gli3," Oncogene, vol. 37, no. 17, pp. 23132325, 2018.

[66] H. Kuroda, N. Kurio, T. Shimo et al., "Oral squamous cell carcinoma-derived sonic Hedgehog promotes angiogenesis," Anticancer Research, vol. 37, no. 12, pp. 6731-6737, 2017. 
[67] K. Wang, D. Chen, Z. Qian, D. Cui, L. Gao, and M. Lou, "Hedgehog/Gli1 signaling pathway regulates MGMT expression and chemoresistance to temozolomide in human glioblastoma," Cancer Cell International, vol. 17, no. 1, p. 117, 2017.

[68] X. Song, L. Yan, C. Lu et al., "Activation of hedgehog signaling and its association with cisplatin resistance in ovarian epithelial tumors," Oncology Letters, vol. 15, no. 4, pp. 5569-5576, 2018.

[69] D. Amakye, Z. Jagani, and M. Dorsch, "Unraveling the therapeutic potential of the Hedgehog pathway in cancer," Nature Medicine, vol. 19, no. 11, pp. 1410-1422, 2013.

[70] Q. Chen, H. Zhang, M. Wu et al., "Discovery of a potent hedgehog pathway inhibitor capable of activating caspase8dependent apoptosis," Journal of Pharmacological Sciences, vol. 137, no. 3, pp. 256-264, 2018.

[71] V. Clement, P. Sanchez, N. de Tribolet, I. Radovanovic, and A. Ruiz i Altaba, "HEDGEHOG-GLI1 Signaling Regulates Human Glioma Growth, Cancer Stem Cell Self- Renewal, and Tumorigenicity," Current Biology, vol. 17, no. 2, pp. 165-172, 2007.

[72] A. Balic, M. D. Sorensen, S. M. Trabulo et al., "Chloroquine targets pancreatic cancer stem cells via inhibition of CXCR4 and hedgehog signaling," Molecular Cancer Therapeutics, vol. 13, no. 7, pp. 1758-1771, 2014.

[73] Y. Koike, Y. Ohta, W. Saitoh et al., "Anti-cell growth and anti-cancer stem cell activities of the non-canonical hedgehog inhibitor GANT61 in triple-negative breast cancer cells," Breast Cancer, vol. 24, no. 5, pp. 683-693, 2017.

[74] Y. Miyazaki, S. Matsubara, Q. Ding et al., "Efficient elimination of pancreatic cancer stem cells by hedgehog/GLI inhibitor GANT61 in combination with mTOR inhibition," Molecular Cancer, vol. 15, no. 1, p. 49, 2016.

[75] N. Fukushima, Y. Minami, S. Kakiuchi et al., "Small-molecule Hedgehog inhibitor attenuates the leukemia-initiation potential of acute myeloid leukemia cells," Cancer Science, vol. 107, no. 10, pp. 1422-1429, 2016.

[76] H. Clevers, "Wnt/ $\beta$-Catenin Signaling in Development and Disease," Cell, vol. 127, no. 3, pp. 469-480, 2006.

[77] R. T. Moon, A. D. Kohn, G. V. De Ferrari, and A. Kaykas, "WNT and $\beta$-catenin signalling: diseases and therapies," Nature Reviews. Genetics, vol. 5, no. 9, pp. 691-701, 2004.

[78] T. Tammela, F. J. Sanchez-Rivera, N. M. Cetinbas et al., "A Wnt-producing niche drives proliferative potential and progression in lung adenocarcinoma," Nature, vol. 545, no. 7654, pp. 355-359, 2017.

[79] A. Prieur, M. Cappellini, G. Habif et al., "Targeting the Wnt pathway and cancer stem cells with anti-progastrin humanized antibodies as a potential treatment for K-RAS-mutated colorectal cancer," Clinical Cancer Research, vol. 23, no. 17, pp. 5267-5280, 2017.

[80] G. B. Jang, I. S. Hong, R. J. Kim et al., "Wnt/ -Catenin smallmolecule inhibitor CWP232228 preferentially inhibits the growth of breast cancer stem-like cells," Cancer Research, vol. 75, no. 8, pp. 1691-1702, 2015.

[81] R. C. Arend, A. I. Londono-Joshi, A. Gangrade et al., "Niclosamide and its analogs are potent inhibitors of $\mathrm{Wnt} / \beta$-catenin, mTOR and STAT3 signaling in ovarian cancer," Oncotarget, vol. 7, no. 52, pp. 86803-86815, 2016.

[82] R. R. Stine and E. L. Matunis, "JAK-STAT signaling in stem cells," in Transcriptional and Translational Regulation of
Stem Cells, G. Hime and H. Abud, Eds., vol. 786 of Advances in Experimental Medicine and Biology, pp. 247-267, Springer, Dordrecht, 2013.

[83] L. Leng, X. Zhong, G. Sun, W. Qiu, and L. Shi, "Demethoxycurcumin was superior to temozolomide in the inhibition of the growth of glioblastoma stem cells in vivo," Tumor Biology, vol. 37, no. 12, pp. 15847-15857, 2016.

[84] P. Kroon, P. A. Berry, M. J. Stower et al., "JAK-STAT blockade inhibits tumor initiation and clonogenic recovery of prostate cancer stem-like cells," Cancer Research, vol. 73, no. 16, pp. 5288-5298, 2013.

[85] M. Y. Yang, H. T. Lee, C. M. Chen, C. C. Shen, and H. I. Ma, "Celecoxib suppresses the phosphorylation of STAT3 protein and can enhance the radiosensitivity of medulloblastoma-derived cancer stem-like cells," International Journal of Molecular Sciences, vol. 15, no. 6, pp. 11013-11029, 2014.

[86] A. Alisi, W. C. Cho, F. Locatelli, and D. Fruci, "Multidrug resistance and cancer stem cells in neuroblastoma and hepatoblastoma," International Journal of Molecular Sciences, vol. 14, no. 12, pp. 24706-24725, 2013.

[87] L. Chen, L. Shi, W. Wang, and Y. Zhou, "ABCG2 downregulation in glioma stem cells enhances the therapeutic efficacy of demethoxycurcumin," Oncotarget, vol. 8, no. 26, pp. 43237-43247, 2017.

[88] Y. Shishido, S. Ueno, R. Yamazaki, M. Nagaoka, and T. Matsuzaki, "ABCG2 inhibitor YHO-13351 sensitizes cancer stem/initiating-like side population cells to irinotecan," Anticancer Research, vol. 33, no. 4, pp. 1379-1386, 2013.

[89] H. Chen, K. Shien, K. Suzawa et al., "Elacridar, a thirdgeneration $\mathrm{ABCB} 1$ inhibitor, overcomes resistance to docetaxel in non-small cell lung cancer," Oncology Letters, vol. 14, no. 4, pp. 4349-4354, 2017.

[90] S. A. Balaji, N. Udupa, M. R. Chamallamudi, V. Gupta, and A. Rangarajan, "Role of the drug transporter $\mathrm{ABCC} 3$ in breast cancer chemoresistance," PLoS One, vol. 11, no. 5, article e0155013, 2016.

[91] C. L. Dai, A. K. Tiwari, C. P. Wu et al., "Lapatinib (Tykerb, GW572016) reverses multidrug resistance in cancer cells by inhibiting the activity of ATP-binding cassette subfamily B member 1 and G member 2," Cancer Research, vol. 68, no. 19, pp. 7905-7914, 2008.

[92] J. Zhao, "Cancer stem cells and chemoresistance: the smartest survives the raid," Pharmacology \& Therapeutics, vol. 160, pp. 145-158, 2016.

[93] R. Januchowski, K. Wojtowicz, and M. Zabel, "The role of aldehyde dehydrogenase (ALDH) in cancer drug resistance," Biomedicine \& Pharmacotherapy, vol. 67, no. 7, pp. 669-680, 2013.

[94] C. Canino, Y. Luo, P. Marcato, G. Blandino, H. I. Pass, and M. Cioce, "A STAT3-NFkB/DDIT3/CEBP $\beta$ axis modulates ALDH1A3 expression in chemoresistant cell subpopulations," Oncotarget, vol. 6, no. 14, pp. 1263712653, 2015.

[95] Z. Kozovska, A. Patsalias, V. Bajzik et al., "ALDH1A inhibition sensitizes colon cancer cells to chemotherapy," $B M C$ Cancer, vol. 18, no. 1, p. 656, 2018.

[96] K. Takahashi and S. Yamanaka, "Induction of pluripotent stem cells from mouse embryonic and adult fibroblast cultures by defined factors," Cell, vol. 126, no. 4, pp. 663-676, 2006. 
[97] A. Doi, I. H. Park, B. Wen et al., "Differential methylation of tissue- and cancer-specific CpG island shores distinguishes human induced pluripotent stem cells, embryonic stem cells and fibroblasts," Nature Genetics, vol. 41, no. 12, pp. 13501353, 2009.

[98] K. Lapinska, G. Faria, S. McGonagle, K. M. Macumber, S. Heerboth, and S. Sarkar, "Cancer progenitor cells: the result of an epigenetic event?," Anticancer Research, vol. 38, no. 1, pp. 1-6, 2018.

[99] G. Singovski, C. Bernal, M. Kuciak, I. Siegl-Cachedenier, A. Conod, and I. A. A. Ruiz, "In vivo epigenetic reprogramming of primary human colon cancer cells enhances metastases," Journal of Molecular Cell Biology, vol. 8, no. 2, pp. 157-173, 2016.

[100] E. N. Wainwright and P. Scaffidi, "Epigenetics and cancer stem cells: unleashing, hijacking, and restricting cellular plasticity," Trends in Cancer, vol. 3, no. 5, pp. 372-386, 2017.

[101] J. Xun, D. Wang, L. Shen et al., "JMJD3 suppresses stem celllike characteristics in breast cancer cells by downregulation of Oct4 independently of its demethylase activity," Oncotarget, vol. 8, no. 13, pp. 21918-21929, 2017.

[102] J. Tian, S. O. Lee, L. Liang et al., "Targeting the unique methylation pattern of androgen receptor (AR) promoter in prostate stem/progenitor cells with 5 -aza-2' -deoxycytidine (5-AZA) leads to suppressed prostate tumorigenesis," Journal of Biological Chemistry, vol. 287, no. 47, pp. 39954-39966, 2012.

[103] S. Y. Li, R. Sun, H. X. Wang et al., "Combination therapy with epigenetic-targeted and chemotherapeutic drugs delivered by nanoparticles to enhance the chemotherapy response and overcome resistance by breast cancer stem cells," Journal of Controlled Release, vol. 205, pp. 7-14, 2015.

[104] Y. Wang, H. Cardenas, F. Fang et al., "Epigenetic targeting of ovarian cancer stem cells," Cancer Research, vol. 74, no. 17, pp. 4922-4936, 2014.

[105] M. C. Manara, S. Valente, C. Cristalli et al., "A quinolinebased DNA methyltransferase inhibitor as a possible adjuvant in osteosarcoma therapy," Molecular Cancer Therapeutics, vol. 17, no. 9, pp. 1881-1892, 2018.

[106] L. Travaglini, L. Vian, M. Billi, F. Grignani, and C. Nervi, "Epigenetic reprogramming of breast cancer cells by valproic acid occurs regardless of estrogen receptor status," The International Journal of Biochemistry \& Cell Biology, vol. 41, no. 1, pp. 225-234, 2009.

[107] P. A. Marks, "Discovery and development of SAHA as an anticancer agent," Oncogene, vol. 26, no. 9, pp. 13511356, 2007.

[108] M. T. Chiao, W. Y. Cheng, Y. C. Yang, C. C. Shen, and J. L. Ko, "Suberoylanilide hydroxamic acid (SAHA) causes tumor growth slowdown and triggers autophagy in glioblastoma stem cells," Autophagy, vol. 9, no. 10, pp. 1509-1526, 2013.

[109] X. Lan, D. J. Jorg, F. M. G. Cavalli et al., "Fate mapping of human glioblastoma reveals an invariant stem cell hierarchy," Nature, vol. 549, no. 7671, pp. 227-232, 2017.

[110] I. San-Millan and G. A. Brooks, "Reexamining cancer metabolism: lactate production for carcinogenesis could be the purpose and explanation of the Warburg Effect," Carcinogenesis, vol. 38, no. 2, pp. 119-133, 2017.

[111] K. Parmar, P. Mauch, J. A. Vergilio, R. Sackstein, and J. D. Down, "Distribution of hematopoietic stem cells in the bone marrow according to regional hypoxia," Proceedings of the National Academy of Sciences of the United States of America, vol. 104, no. 13, pp. 5431-5436, 2007.

[112] T. Suda, K. Takubo, and G. L. Semenza, "Metabolic regulation of hematopoietic stem cells in the hypoxic niche," Cell Stem Cell, vol. 9, no. 4, pp. 298-310, 2011.

[113] W. Zhou, M. Choi, D. Margineantu et al., "HIF1 $\alpha$ induced switch from bivalent to exclusively glycolytic metabolism during ESC-to-EpiSC/hESC transition," The EMBO Journal, vol. 31, no. 9, pp. 2103-2116, 2012.

[114] A. Prigione, B. Fauler, R. Lurz, H. Lehrach, and J. Adjaye, "The senescence-related mitochondrial/oxidative stress pathway is repressed in human induced pluripotent stem cells," Stem Cells, vol. 28, no. 4, pp. 721-733, 2010.

[115] P. P. Liu, J. Liao, Z. J. Tang et al., "Metabolic regulation of cancer cell side population by glucose through activation of the Akt pathway," Cell Death \& Differentiation, vol. 21, no. 1, pp. 124-135, 2014.

[116] W. Feng, A. Gentles, R. V. Nair et al., "Targeting unique metabolic properties of breast tumor initiating cells," Stem Cells, vol. 32, no. 7, pp. 1734-1745, 2014.

[117] W. Chen, J. Dong, J. Haiech, M. C. Kilhoffer, and M. Zeniou, "Cancer stem cell quiescence and plasticity as major challenges in cancer therapy," Stem Cells International, vol. 2016, Article ID 1740936, 16 pages, 2016.

[118] Q. Xu, Q. Zhang, Y. Ishida et al., "EGF induces epithelialmesenchymal transition and cancer stem-like cell properties in human oral cancer cells via promoting Warburg effect," Oncotarget, vol. 8, no. 6, pp. 9557-9571, 2017.

[119] A. Janzer, N. J. German, K. N. Gonzalez-Herrera, J. M. Asara, M. C. Haigis, and K. Struhl, "Metformin and phenformin deplete tricarboxylic acid cycle and glycolytic intermediates during cell transformation and NTPs in cancer stem cells," Proceedings of the National Academy of Sciences of the United States of America, vol. 111, no. 29, pp. 10574-10579, 2014.

[120] Y. Ji, C. Yang, Z. Tang et al., "Adenylate kinase hCINAP determines self-renewal of colorectal cancer stem cells by facilitating LDHA phosphorylation," Nature Communications, vol. 8, article 15308, 2017.

[121] E. D. Lagadinou, A. Sach, K. Callahan et al., "BCL-2 inhibition targets oxidative phosphorylation and selectively eradicates quiescent human leukemia stem cells," Cell Stem Cell, vol. 12, no. 3, pp. 329-341, 2013.

[122] A. Pasto, C. Bellio, G. Pilotto et al., "Cancer stem cells from epithelial ovarian cancer patients privilege oxidative phosphorylation, and resist glucose deprivation," Oncotarget, vol. 5, no. 12, pp. 4305-4319, 2014.

[123] C. W. Song, H. Lee, R. P. Dings et al., "Metformin kills and radiosensitizes cancer cells and preferentially kills cancer stem cells," Scientific Reports, vol. 2, no. 1, article 362, 2012 .

[124] M. Fiorillo, R. Lamb, H. B. Tanowitz et al., "Repurposing atovaquone: targeting mitochondrial complex III and OXPHOS to eradicate cancer stem cells," Oncotarget, vol. 7, no. 23, pp. 34084-34099, 2016.

[125] B. Ozsvari, G. Bonuccelli, R. Sanchez-Alvarez, R. Foster, F. Sotgia, and M. P. Lisanti, "Targeting flavin-containing enzymes eliminates cancer stem cells (CSCs), by inhibiting mitochondrial respiration: Vitamin B2 (Riboflavin) in cancer therapy," Aging, vol. 9, no. 12, pp. 2610-2628, 2017. 
[126] T. Relation, M. Dominici, and E. M. Horwitz, "Concise review: an (im)penetrable shield: how the tumor microenvironment protects cancer stem cells," Stem Cells, vol. 35, no. 5, pp. 1123-1130, 2017.

[127] A. D. Theocharis, S. S. Skandalis, C. Gialeli, and N. K. Karamanos, "Extracellular matrix structure," Advanced Drug Delivery Reviews, vol. 97, pp. 4-27, 2016.

[128] N. N. Rahbari, D. Kedrin, J. Incio et al., "Anti-VEGF therapy induces ECM remodeling and mechanical barriers to therapy in colorectal cancer liver metastases," Science Translational Medicine, vol. 8, no. 360, article 360ra135, 2016.

[129] C. H. C. Sukowati, B. Anfuso, E. Fiore et al., "Hyaluronic acid inhibition by 4 -methylumbelliferone reduces the expression of cancer stem cells markers during hepatocarcinogenesis," Scientific Reports, vol. 9, no. 1, article 4026, 2019.

[130] M. Prieto-Vila, R. U. Takahashi, W. Usuba, I. Kohama, and T. Ochiya, "Drug resistance driven by cancer stem cells and their niche," International Journal of Molecular Sciences, vol. 18, no. 12, article 2574, 2017.

[131] N. Nair, A. S. Calle, M. H. Zahra et al., "A cancer stem cell model as the point of origin of cancer-associated fibroblasts in tumor microenvironment," Scientific Reports, vol. 7, no. 1, article 6838, 2017.

[132] G. Valenti, H. M. Quinn, G. Heynen et al., "Cancer stem cells regulate cancer-associated fibroblasts via activation of Hedgehog signaling in mammary gland tumors," Cancer Research, vol. 77, no. 8, pp. 2134-2147, 2017.

[133] A. L. Strong, J. F. Ohlstein, B. A. Biagas et al., "Leptin produced by obese adipose stromal/stem cells enhances proliferation and metastasis of estrogen receptor positive breast cancers," Breast Cancer Research, vol. 17, no. 1, article 112, 2015.

[134] A. L. Strong, T. A. Strong, L. V. Rhodes et al., "Obesity associated alterations in the biology of adipose stem cells mediate enhanced tumorigenesis by estrogen dependent pathways," Breast Cancer Research, vol. 15, no. 5, article R102, 2013.

[135] P. A. Zuk, M. Zhu, H. Mizuno et al., "Multilineage cells from human adipose tissue: implications for cell-based therapies," Tissue Engineering, vol. 7, no. 2, pp. 211-228, 2001.

[136] R. A. Sabol, A. Beighley, P. Giacomelli et al., "Obesityaltered adipose stem cells promote $\mathrm{ER}^{+}$breast cancer metastasis through estrogen independent pathways," International Journal of Molecular Sciences, vol. 20, no. 6, p. 1419, 2019.

[137] T. Lindstad, S. Qu, J. Sikkeland et al., "STAMP2 is required for human adipose-derived stem cell differentiation and adipocyte-facilitated prostate cancer growth in vivo," Oncotarget, vol. 8, no. 54, pp. 91817-91827, 2017.

[138] M. G. Scioli, S. Artuso, C. D'Angelo et al., "Adipose-derived stem cell-mediated paclitaxel delivery inhibits breast cancer growth," PLoS One, vol. 13, no. 9, article e0203426, 2018.

[139] K. D. Tang, J. Liu, L. Jovanovic et al., “Adipocytes promote prostate cancer stem cell self-renewal through amplification of the cholecystokinin autocrine loop," Oncotarget, vol. 7, no. 4, pp. 4939-4948, 2016.

[140] A. G. Via, A. Frizziero, and F. Oliva, "Biological properties of mesenchymal Stem Cells from different sources," Muscles Ligaments and Tendons Journal, vol. 2, no. 3, pp. 154162, 2012.
[141] P. M. Aponte and A. Caicedo, "Stemness in cancer: stem cells, cancer stem cells, and their microenvironment," Stem Cells International, vol. 2017, Article ID 5619472, 17 pages, 2017.

[142] J. M. Roodhart, L. G. Daenen, E. C. Stigter et al., "Mesenchymal stem cells induce resistance to chemotherapy through the release of platinum-induced fatty acids," Cancer Cell, vol. 20, no. 3, pp. 370-383, 2011.

[143] S. Krishnamurthy, Z. Dong, D. Vodopyanov et al., "Endothelial cell-initiated signaling promotes the survival and selfrenewal of cancer stem cells," Cancer Research, vol. 70, no. 23, pp. 9969-9978, 2010.

[144] N. Charles, T. Ozawa, M. Squatrito et al., "Perivascular nitric oxide activates notch signaling and promotes stemlike character in PDGF-induced glioma cells," Cell Stem Cell, vol. 6, no. 2, pp. 141-152, 2010.

[145] S. Palmer, L. Albergante, C. C. Blackburn, and T. J. Newman, "Thymic involution and rising disease incidence with age," Proceedings of the National Academy of Sciences of the United States of America, vol. 115, no. 8, pp. 1883-1888, 2018.

[146] Y. Kokubu, K. Tabu, N. Muramatsu et al., "Induction of protumoral CD $11 \mathrm{c}^{\text {high }}$ macrophages by glioma cancer stem cells through GM-CSF," Genes to Cells, vol. 21, no. 3, pp. 241-251, 2016.

[147] Y. Lee, J. H. Shin, M. Longmire et al., "CD44 ${ }^{+}$cells in head and neck squamous cell carcinoma suppress t-cell-mediated immunity by selective constitutive and inducible expression of PD-L1," Clinical Cancer Research, vol. 22, no. 14, pp. 3571-3581, 2016.

[148] I. Bozic and M. A. Nowak, "Timing and heterogeneity of mutations associated with drug resistance in metastatic cancers," Proceedings of the National Academy of Sciences of the United States of America, vol. 111, no. 45, pp. 15964-15968, 2014.

[149] M. A. Locatelli, P. Aftimos, E. C. Dees et al., "Phase I study of the gamma secretase inhibitor PF-03084014 in combination with docetaxel in patients with advanced triple-negative breast cancer," Oncotarget, vol. 8, no. 2, pp. 2320-2328, 2017.

[150] M. J. McKeage, D. Kotasek, B. Markman et al., "Phase IB trial of the anti-cancer stem cell DLL4-binding agent demcizumab with pemetrexed and carboplatin as first-line treatment of metastatic non-squamous NSCLC," Targeted Oncology, vol. 13, no. 1, pp. 89-98, 2018.

[151] A. F. Schott, L. J. Goldstein, M. Cristofanilli et al., "Phase Ib pilot study to evaluate reparixin in combination with weekly paclitaxel in patients with HER-2-negative metastatic breast cancer," Clinical Cancer Research, vol. 23, no. 18, pp. 53585365, 2017.

[152] M. Baumann, M. Krause, and R. Hill, "Exploring the role of cancer stem cells in radioresistance," Nature Reviews Cancer, vol. 8, no. 7, pp. 545-554, 2008.

[153] X. Chen, R. Liao, D. Li, and J. Sun, "Induced cancer stem cells generated by radiochemotherapy and their therapeutic implications," Oncotarget, vol. 8, no. 10, pp. 17301-17312, 2017.

[154] K. Ogawa, Y. Yoshioka, F. Isohashi, Y. Seo, K. Yoshida, and H. Yamazaki, "Radiotherapy targeting cancer stem cells: current views and future perspectives," Anticancer Research, vol. 33, no. 3, pp. 747-754, 2013.

[155] S. De, D. J. Lindner, C. J. Coleman, G. Wildey, A. Dowlati, and G. R. Stark, "The FACT inhibitor CBL0137 synergizes with cisplatin in small-cell lung cancer by Increasing 
NOTCH1 Expression and targeting tumor-initiating cells," Cancer Research, vol. 78, no. 9, pp. 2396-2406, 2018.

[156] L. MacDonagh, S. G. Gray, E. Breen et al., "BBI608 inhibits cancer stemness and reverses cisplatin resistance in NSCLC," Cancer Letters, vol. 428, pp. 117-126, 2018.

[157] H. Noh, Q. Zhao, J. Yan et al., "Cell surface vimentin-targeted monoclonal antibody $86 \mathrm{C}$ increases sensitivity to temozolomide in glioma stem cells," Cancer Letters, vol. 433, pp. 176-185, 2018.

[158] C. R. Timme, B. H. Rath, J. W. O'Neill, K. Camphausen, and P. J. Tofilon, "The DNA-PK inhibitor VX-984 enhances the radiosensitivity of glioblastoma cells GrownIn Vitroand as orthotopic xenografts," Molecular Cancer Therapeutics, vol. 17, no. 6, pp. 1207-1216, 2018.

[159] P. Lesueur, F. Chevalier, E. A. El-Habr et al., "Radiosensitization effect of talazoparib, a parp inhibitor, on glioblastoma stem cells exposed to low and high linear energy transfer radiation," Scientific Reports, vol. 8, no. 1, article 3664, 2018.

[160] M. A. Macha, S. Rachagani, A. K. Qazi et al., "Afatinib radiosensitizes head and neck squamous cell carcinoma cells by targeting cancer stem cells," Oncotarget, vol. 8, no. 13, pp. 20961-20973, 2017. 




The Scientific World Journal
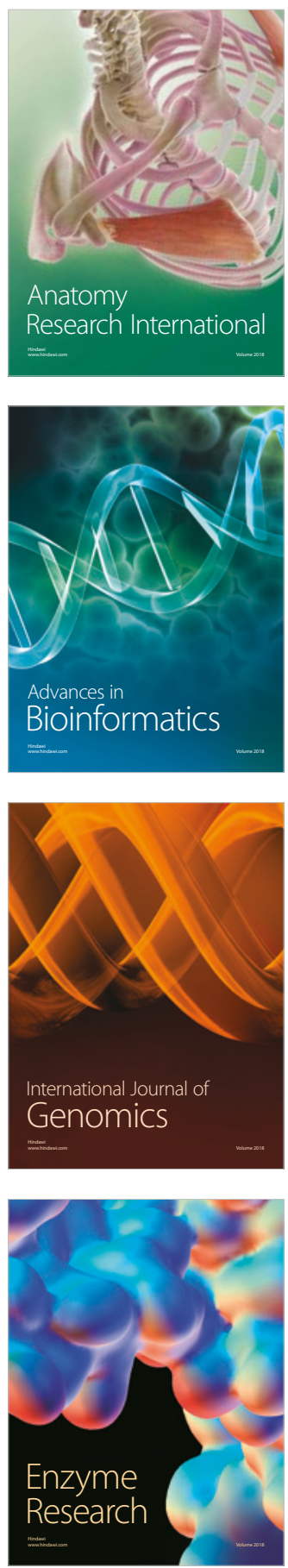
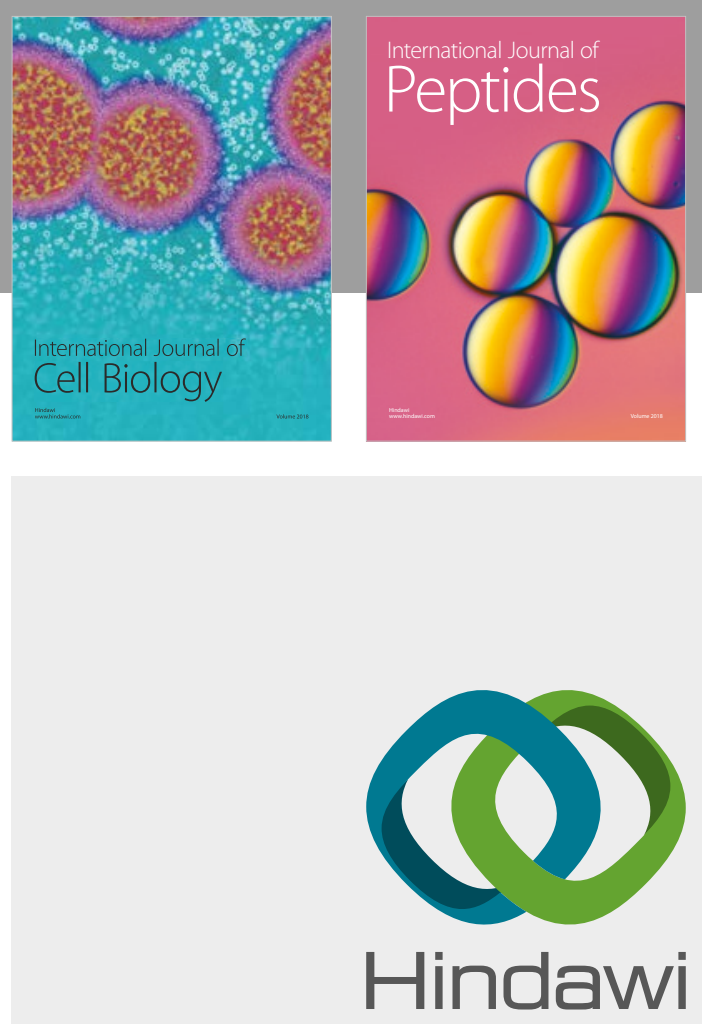

Submit your manuscripts at

www.hindawi.com
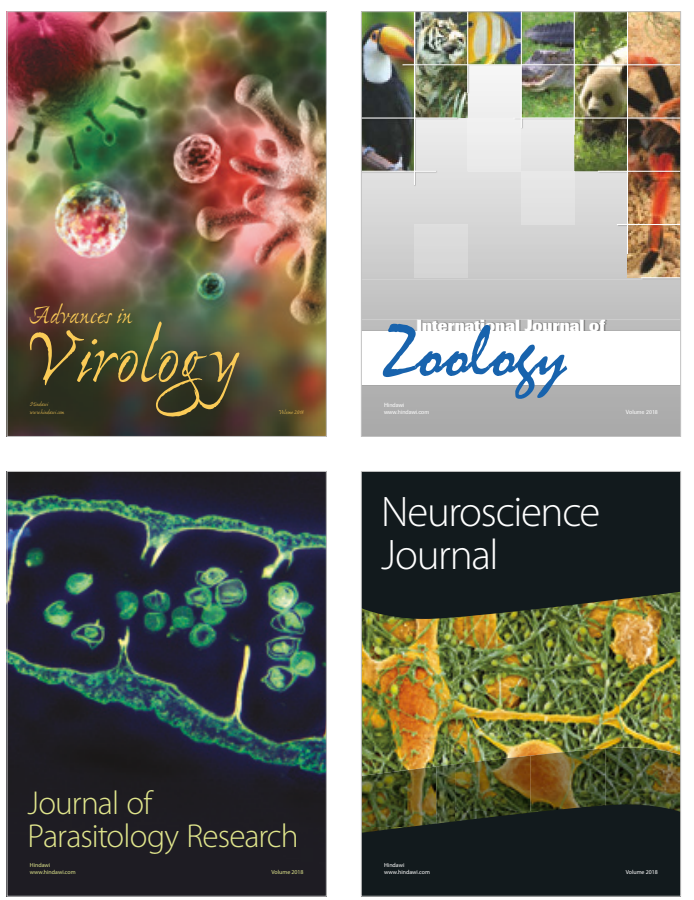
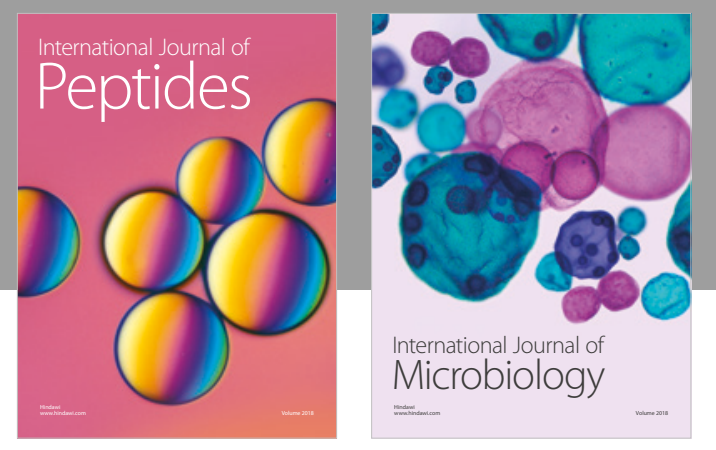

nternational Journal of Microbiology
Journal of
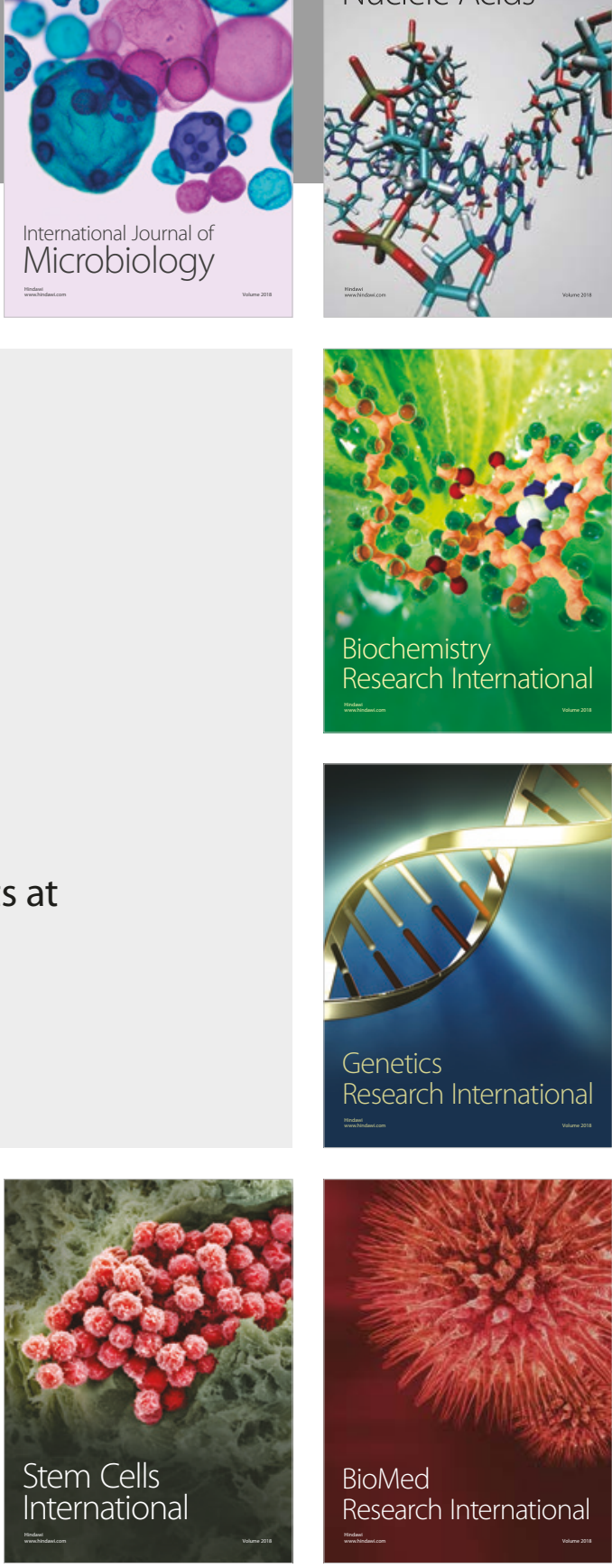
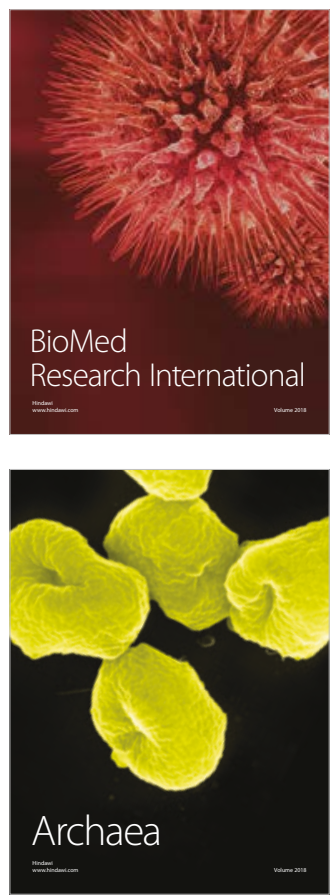